Louisiana State University

LSU Digital Commons

$1-1-2014$

\title{
Kepler eclipsing binary stars. V. Identification of 31 candidate eclipsing binaries in the $\mathrm{K} 2$ engineering dataset
}

\author{
Kyle E. Conroy \\ Vanderbilt University \\ Andrej Prša \\ Villanova University \\ Keivan G. Stassun \\ Vanderbilt University \\ Steven Bloemen \\ Radboud University Nijmegen \\ Mahmoud Parvizi \\ Vanderbilt University
}

See next page for additional authors

Follow this and additional works at: https://digitalcommons.Isu.edu/physics_astronomy_pubs

\section{Recommended Citation}

Conroy, K., Prša, A., Stassun, K., Bloemen, S., Parvizi, M., Quarles, B., Boyajian, T., Barclay, T., Shporer, A., Latham, D., \& Abdul-Masih, M. (2014). Kepler eclipsing binary stars. V. Identification of 31 candidate eclipsing binaries in the K2 engineering dataset. Publications of the Astronomical Society of the Pacific, 126 (944), 914-922. https://doi.org/10.1086/678953

This Article is brought to you for free and open access by the Department of Physics \& Astronomy at LSU Digital Commons. It has been accepted for inclusion in Faculty Publications by an authorized administrator of LSU Digital Commons. For more information, please contact ir@lsu.edu. 


\section{Authors}

Kyle E. Conroy, Andrej Prša, Keivan G. Stassun, Steven Bloemen, Mahmoud Parvizi, Billy Quarles, Tabetha Boyajian, Thomas Barclay, Avi Shporer, David W. Latham, and Michael Abdul-Masih 


\section{CHICAGO JOURNALS}

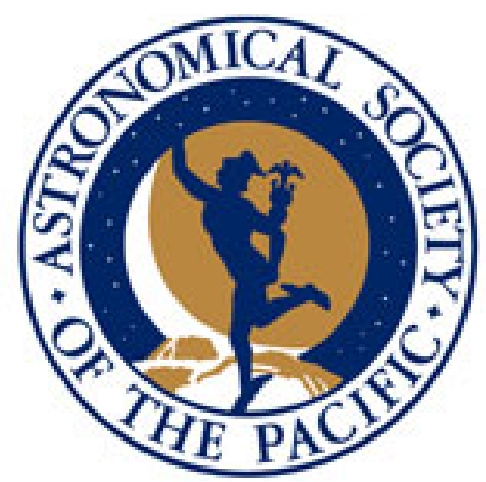

Kepler Eclipsing Binary Stars. V. Identification of 31 Candidate Eclipsing Binaries in the K2 Engineering Dataset

Author(s): Kyle E. Conroy, Andrej Prša, Keivan G. Stassun, Steven Bloemen, Mahmoud Parvizi, Billy Quarles, Tabetha Boyajian, Thomas Barclay, Avi Shporer, David W. Latham, and Michael Abdul-Masih

Source: Publications of the Astronomical Society of the Pacific, Vol. 126, No. 944 (October 2014), pp. 914-922

Published by: The University of Chicago Press on behalf of the Astronomical Society of the Pacific Stable URL: http://www.jstor.org/stable/10.1086/678953

Accessed: 11/12/2014 11:01

Your use of the JSTOR archive indicates your acceptance of the Terms \& Conditions of Use, available at http://www.jstor.org/page/info/about/policies/terms.jsp

JSTOR is a not-for-profit service that helps scholars, researchers, and students discover, use, and build upon a wide range of content in a trusted digital archive. We use information technology and tools to increase productivity and facilitate new forms of scholarship. For more information about JSTOR, please contact support@ jstor.org. 


\title{
Kepler Eclipsing Binary Stars. V. Identification of 31 Candidate Eclipsing Binaries in the K2 Engineering Dataset
}

\author{
Kyle E. Conroy, ${ }^{1}$ Andrej Prša, ${ }^{2}$ Keivan G. Stassun,${ }^{1,3}$ Steven Bloemen,${ }^{4}$ Mahmoud Parvizi, ${ }^{1}$ Billy Quarles, ${ }^{5}$ \\ Tabetha Boyajian, ${ }^{6}$ Thomas Barclay, ${ }^{5,7}$ Avi Shporer, ${ }^{8,9}$ David W. Latham, ${ }^{10}$ And Michael Abdul-Masih ${ }^{2}$ \\ Received 2014 July 14; accepted 2014 September 05; published 2014 October 2
}

\begin{abstract}
Over 2500 eclipsing binaries were identified and characterized from the ultraprecise photometric data provided by the Kepler space telescope. Kepler is now beginning its second mission, K2, which is proving to again provide ultraprecise photometry for a large sample of eclipsing binary stars. In the 1951 light curves covering 12 days in the $\mathrm{K} 2$ engineering dataset, we have identified and determined the ephemerides for 31 candidate eclipsing binaries that demonstrate the capabilities for eclipsing binary science in the upcoming campaigns in K2. Of those, 20 are new discoveries. We describe both manual and automated approaches to harvesting the complete set of eclipsing binaries in the $\mathrm{K} 2$ data, provide identifications and details for the full set of candidate eclipsing binaries present in the engineering dataset, and discuss the prospects for application of eclipsing binary searches in the K2 mission.
\end{abstract}

\section{INTRODUCTION}

The Kepler satellite (Batalha et al. 2010) observed over 150,000 stars in its original mission, which acquired over $4 \mathrm{yr}$ of high-precision photometry. This dataset was followed by a large effort to study the eclipsing binary (EB) population in the Kepler field, resulting in the detection and characterization of over 2500 EB stars (Prša et al. 2011; Slawson et al. 2011), the measurements of eclipse timing variations (Conroy et al. 2014), and the discovery of several circumbinary planets (Doyle et al. 2011; Welsh et al. 2012).

Now that Kepler has transitioned to its repurposed mission, $\mathrm{K} 2$, it is providing 80 days of continuous high-precision photometry across each of 10 fields in the ecliptic plane, once again giving great scientific opportunity to identify and characterize EBs (Prša et al. 2014). Although the photometric precision

\footnotetext{
${ }^{1}$ Department of Physics and Astronomy, Vanderbilt University, Nashville, TN 37235 .

${ }^{2}$ Department of Astrophysics and Planetary Sciences, Villanova University, Villanova, PA 19085.

${ }^{3}$ Department of Physics, Fisk University, Nashville, TN 37208.

${ }^{4}$ Department of Astrophysics, Institute for Mathematics, Astrophysics, and Particle Physics, Radboud University Nijmegen, PO Box 9010, 6500 GL, Nijmegen, The Netherlands. Also at: Instituut voor Sterrenkunde, KU Leuven, Celestijnenlaan 200D, B-3001 Heverlee, Belgium.

${ }^{5}$ NASA Ames Research Center, M/S 244-30, Moffett Field, CA 94035.

${ }^{6}$ Department of Astronomy, Yale University, New Haven, CT 06511.

${ }^{7}$ Bay Area Environmental Research Institute, 596 1st Street West, Sonoma, CA 95476

${ }^{8}$ Division of Geological and Planetary Sciences, California Institute of Technology, Pasadena, CA 91125. Also at: Jet Propulsion Laboratory, California Institute of Technology, Pasadena, CA 91109.

${ }^{9}$ NASA Sagan Fellow.

${ }^{10}$ Harvard-Smithsonian Center for Astrophysics, 60 Garden Street, Cambridge, MA 02138 .
}

compared to the original Kepler mission is expected to be slightly lower due to a decrease in pointing accuracy, $\mathrm{K} 2$ is still expected to obtain data an order of magnitude better than is possible from the ground. With the upcoming Transiting Exoplanet Survey Satellite (TESS) mission, EBs identified in K2 will become prime targets for further follow-up, allowing us to extend the time baseline and continue searching for triple systems (stellar and substellar) through eclipse timing variations and searching for transiting events.

Nonetheless, it is important to assess both the potential and the challenges of harvesting EBs from the new K2 data. In this paper, we utilize the first publicly available dataset from K2the engineering dataset-to perform a cursory look at the EB identification methods, as applied to K2. In $\S 2$, we describe the K2 data that we use and the data-level processing of the K2 light curves. In $\S 3$, we present the manual and automated methods that we employ to identify and classify candidate EBs in the $\mathrm{K} 2$ dataset, along with their ephemerides. We conclude in $\S 4$ with a brief summary and a brief discussion of prospects for $\mathrm{EB}$ science in the full upcoming $\mathrm{K} 2$ mission.

\section{K2 DATA AND PROCESSING}

Unlike the main Kepler mission that focused on a predetermined set of targets within the fixed field of view, the targets for each K2 campaign are solicited from the community, with $\sim 10,000$ long-cadence (29.43 minute) and $\sim 100$ short-cadence (54.2 s) targets selected for observations from each field (Howell et al. 2014). The Kepler Eclipsing Binary Working Group contributes a selection of science targets based on a cross-check of all objects in each $\mathrm{K} 2$ campaign field with available variable and binary star catalogs. One hundred and sixty 
four of 7757 targets selected for observation in campaign 0 and 49 of 21,647 targets in campaign 1 were preidentified as EBs.

In the engineering dataset, there are a total of 1951 longcadence objects observed, in addition to 128 engineering apertures. Data were observed in a cadence of 30 minutes and spanning a total of 12 days. These targets were not solicited; instead, they have been selected randomly, for the purpose of engineering evaluation.

\subsection{LIGHT CURVE EXTRACTION FROM PIXEL DATA}

For the engineering run of $\mathrm{K} 2$, only calibrated pixel data were made available, in contrast to the datasets released for the original mission, which also included extracted light curves. For this work, we have extracted light curves for all 1951 targets from the pixel data, using the tools used and presented in, e.g., Pápics et al. (2013). We have removed the background flux in the pixels using a low-order spline fit to all available pixels around the targets. The light curves were then constructed by adding up all flux in the pixels around the central pixel that have more than 100 counts as their 99.9 percentile value for flux. This creates a constant mask that includes all pixels that ever,

TABLE 1

MASKS fOR CANDIDATE ECLIPSING BINARIES IN K2

\begin{tabular}{|c|c|c|}
\hline Kepler identification & Pixels in mask & Std around polynomial fit \\
\hline 60017809 & 73 & 0.0052 \\
\hline 60017810 & 19 & 0.0468 \\
\hline 60017812 & 39 & 0.0144 \\
\hline 60017814 & 94 & 0.0072 \\
\hline 60017815 & 57 & 0.0072 \\
\hline 60017816 & 29 & 0.0277 \\
\hline 60017821 & 59 & 0.0078 \\
\hline 60017822 & 76 & 0.0084 \\
\hline 60017887 & 115 & 0.0020 \\
\hline 60017946 & 4 & 0.0287 \\
\hline 60017969 & 6 & 0.0640 \\
\hline 60017970 & 28 & 0.0075 \\
\hline 60018031 & 8 & 0.0100 \\
\hline 60018081 & 51 & 0.0027 \\
\hline 60018229 & 103 & 0.0050 \\
\hline 60018241 & 49 & 0.0072 \\
\hline 60018243 & 78 & 0.0032 \\
\hline 60018343 & 106 & 0.0016 \\
\hline 60018394 & 93 & 0.0015 \\
\hline 60018435 & 104 & 0.0063 \\
\hline 60019244 & 26 & 0.0228 \\
\hline 60019950 & 27 & 0.0030 \\
\hline 60020058 & 267 & 0.0027 \\
\hline 60020298 & 39 & 0.0162 \\
\hline 60021220 & 20 & 0.0122 \\
\hline 60021491 & 73 & 0.0056 \\
\hline 60021545 & 84 & 0.0006 \\
\hline 60023653 & 72 & 0.0218 \\
\hline 60024040 & 58 & 0.0023 \\
\hline 60024244 & 63 & 0.0135 \\
\hline 60024523 & 62 & 0.0023 \\
\hline
\end{tabular}

with the exception of outliers, contribute significant flux. The number of pixels in the masks for each EB is listed in Table 1. We find this to be a near-optimal choice, given that including pixels with less flux would increase the noise, and limiting the pixel selection to pixels with higher count levels would increase systematic trends.

The extracted light curves are detrended to remove any trends, instrumental or astrophysical, not related to the EB signal. This is done using an iterative sigma-clipping technique to divide the light curve by a polynomial fitted to the baseline of the data (see Prša et al. [2011] for details). In addition, the first 2 days of engineering data are particularly noisy for many of the targets, likely due to instrumental and satellite pointing issues, and were removed from all subsequent analysis (see also Vanderburg \& Johnson 2014).

\subsection{Noise Properties of the K2 Engineering Light Curves}

The scatter for each of the extracted 1951 light curves as a function of pixel mask size and magnitude is shown in Figures 1 and 2, respectively. The candidate EBs identified in this paper (§ 3) are depicted by square markers, with their sigmas computed about an analytical fit to the EB signal (see § 3.2). As is evident from these figures, the $\mathrm{K} 2$ engineering light curves overall exhibit a strongly bimodal distribution of sigma values, and this bimodal character is largely independent of pixel mask size or stellar magnitude. The reason for this bimodal distribution of light-curve sigmas is not clear.

Within each of the two groupings of stars in these figures, there is the expected trend of decreasing sigma with increasing brightness and increasing pixel mask size. The candidate EBs identified in this paper roughly sample the underlying distribution of light-curve noise properties, however, these do appear to

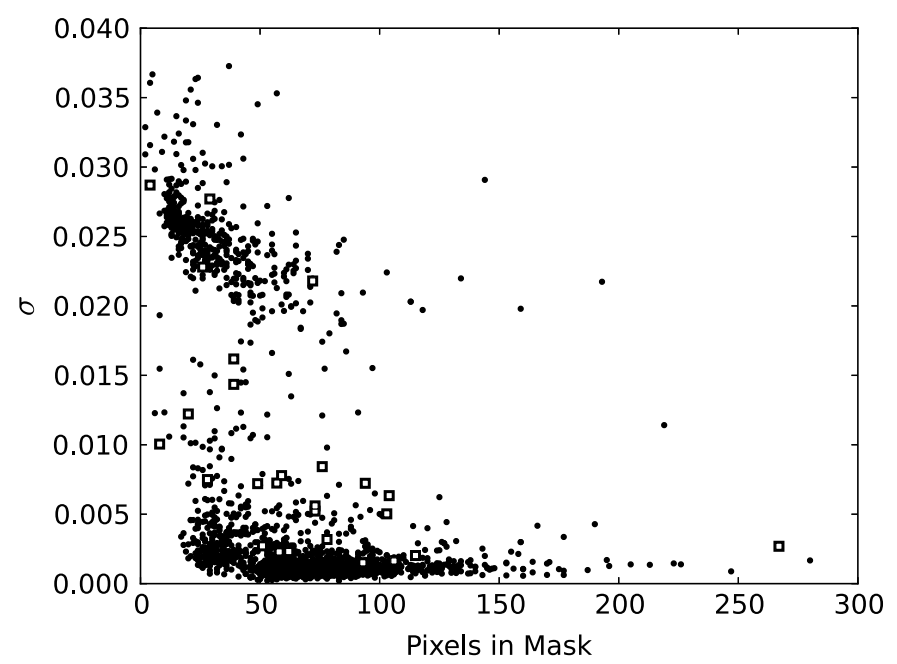

FIG. 1.-Standard deviation of the extracted light curves as a function of the number of pixels in the mask. The values for the EBs listed in this paper are computed based on the residuals around the EB signal and are shown as squares. 


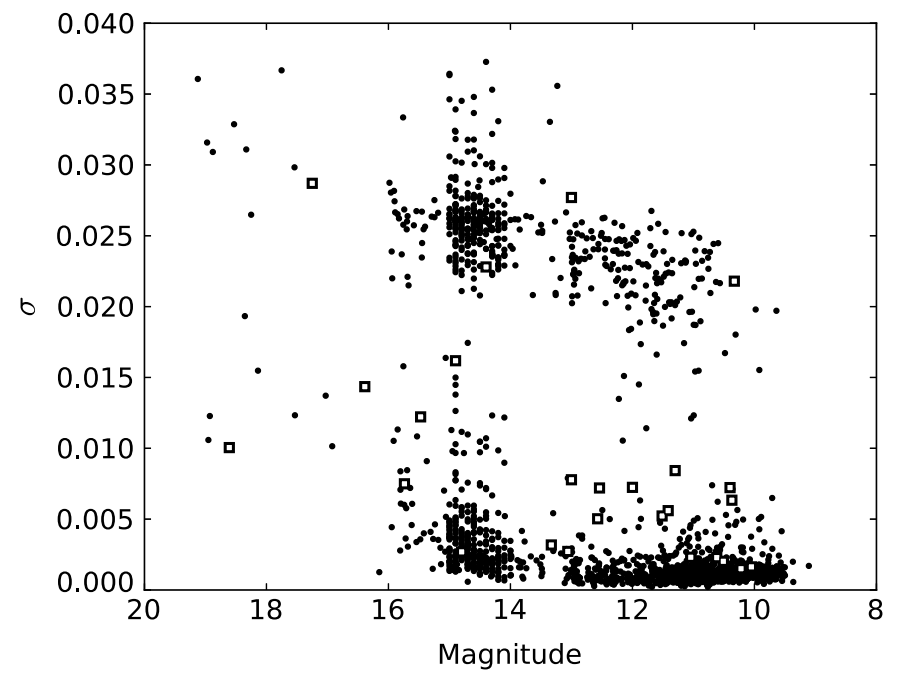

FIG. 2.-Standard deviation of the extracted light curves as a function of the listed Kepler magnitude. The values for the candidate EBs listed in this paper are computed based on the residuals around the EB signal and are shown as squares.

exhibit somewhat higher sigma relative to the other stars of comparable magnitude and pixel mask size, since the analytical models we use to represent them do not fully capture their intrinsic light-curve variations (see $\S 3.2$ ).

A more thorough investigation of the $\mathrm{K} 2$ engineering lightcurve noise properties is beyond the scope of this paper. However, as this paper represents one of the first investigations utilizing the $\mathrm{K} 2$ engineering dataset, the noise properties presented in Figures 1 and 2 may provide the community with a means for estimating the expected light-curve precision of the observed $\mathrm{K} 2$ engineering targets as functions of pixel mask size and magnitude. We note that Vanderburg \& Johnson (2014) have recently reported that improvements in the $\mathrm{K} 2$ photometric precision can be achieved through an optimal, pixel "self flat-fielding" approach. It is possible that the $\mathrm{K} 2$ engineering data noise properties described here can be ameliorated through such an approach.

\section{RESULTS: EBS IN THE K2 ENGINEERING DATASET}

\subsection{Manual EB Identification}

In the K2 engineering target list, nine objects (60017809, 60017810, 60017812, 60017814, 60017815, 60017816, $60017818,60017821,60017822)$ were identified as previously known EBs. One of these (60017818) did not show a clear EB signature in the 12 days of $\mathrm{K} 2$ data, so it was excluded, but the remaining eight were all recovered independently.

Through manual inspection of all 1951 long-cadence light curves, we identified a total of 37 candidate EBs in the K2 engineering dataset. As in the original Kepler dataset, we cannot claim with absolute certainty that these are all true EBs, and some of these signals (e.g., 60017887, 60018031, 60018081, 60019244) could actually be due to pulsating or spotted stars rather than an eclipsing binary or ellipsoidal variable. To confirm their nature as an EB they would likely need to be validated by photometry or radial velocity follow-up, so we instead report these as candidate EBs based on their light-curve characteristics. However, see $\S 3.3$ for evidence from automated classification that these are indeed bona fide EBs.

Some light curves also exhibit signals in addition to the EB signal (either one of the EB members is a variable or a background variable is blending with the EB). Several (e.g., 60018241, 60018343, 60020058, 60024040, 60021220, 60023653 ) show residuals on top of the EB signal, which could be indicative of some other short-period variation. It is difficult to tell whether any of these individual remaining variations are astrophysical, due to some sort of pulsations, or instrumental.

In the original mission, we identified EBs through a variety of methods (Prša et al. 2011), but since there were no threshold crossing events (TCEs-automated detection of transit and eclipse-like features) released by the Kepler science office for the engineering dataset, manually inspecting each light curve was a necessary step in order to test the feasibility of automated detection of eclipsing binary signals in K2 data. Candidate EBs were identified if they showed clear periodic ellipsoidal variation or eclipses in the light curves that repeated at least 3 times in the 12-day baseline of the data. If a light curve showed one or two single eclipse events, the source is included in the list, but ephemerides could not be determined. In the few cases in which only two cycles were observed, the period is still reported if it could be confirmed in the $\sim 2$ days of data that had been trimmed prior to analysis. Additionally, 60018343 is known to be a transiting planet false-positive (Poleski et al. 2010), and so is included despite having a period too long to observe three full cycles. The unphased light curves for the objects can be seen in Figure 3. Planet Hunters ${ }^{11}$ (Fischer et al. 2012) had independently detected and identified several of these EBs as well.

Of these 37 , there were six sets of nearby targets that exhibited the same period and shape in their light curves. It is likely that we are seeing the same EB signal from a single source bleeding into both apertures. Due to the large pixel and aperture sizes, it is difficult to determine the true source of this potential EB signal, and there is no direct mapping from Kepler identification to stellar objects. In these cases, the target with the larger amplitude signal was considered the true source, and the other target was marked as a blend (false-positive) and removed from the list. After removing these cases, there are a total of 31 manually detected candidate EBs (Table 2). The removed targets that were determined to be a duplicate source are listed in Table 3. This only removes the same EB signal from appearing twicewithout follow-up the exact source cannot be confirmed and there may still likely be contamination from other sources in addition to the EB in the aperture.

\footnotetext{
${ }^{11} \mathrm{http}: / /$ www.planethunters.org.
} 


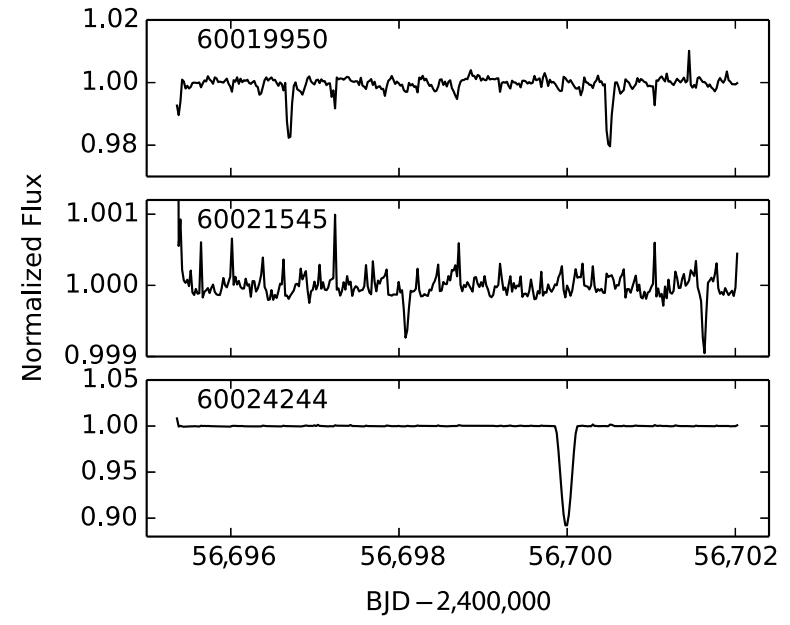

FIG. 3.-Unphased detrended light curves for three identified EBs with unconfirmed periods.
TABLE 3

BLENDED CANDIDATE EBs IN K2

\begin{tabular}{lc}
\hline \hline Kepler identification (EB) & Kepler identification (blend) \\
\hline 60017809 & 60023285 \\
60017815 & 60018240 \\
60017816 & 60042608 \\
60017822 & 60023349 \\
60018081 & 60017828 \\
60024523 & 60024522 \\
\hline
\end{tabular}

The K2 engineering target list, unlike the KIC (Kepler Input Catalog) used for the original Kepler mission and the EPIC (Ecliptic Plane Input Catalog) used for the K2 campaigns, does not include target object names. All identified candidate EBs were cross-matched against known sources by their target coordinates with a radius of $1^{\prime}$. These nearby sources and their previous characterizations are listed in Table 4 . We have thus identified 20 previously unknown EBs as candidate EBs.

Kepler identification 60017806 was also initially identified as a candidate EB, but is actually a known extrasolar planet (WASP-28b) and was removed from the catalog.

TABLE 2

CANDidATE ECLIPSING Binaries IN K2

\begin{tabular}{|c|c|c|c|c|c|c|c|}
\hline Kepler ID & Kepler mag & R.A. (deg) & Decl. (deg) & Morph & Period (days) & $\mathrm{BJD}_{0}-2,400,000$ & EBF confidence \\
\hline 60017809 & 11.51 & 352.388100 & -3.768842 & 0.87 & 0.36369 & $55,001.1374$ & 0.99 \\
\hline 60017810 & 14.53 & 1.157580 & 3.550330 & 0.79 & 0.26151 & $55,000.1673$ & 0.99 \\
\hline 60017812 & 16.39 & 4.170960 & -0.156970 & 0.94 & 0.19858 & $54,999.9260$ & 0.99 \\
\hline 60017814 & 10.40 & 356.826450 & -8.086691 & 0.80 & 0.48146 & $54,998.4955$ & 0.99 \\
\hline 60017815 & 12.00 & 355.528771 & -3.099600 & 0.77 & 0.46341 & $55,000.4769$ & 0.99 \\
\hline 60017816 & 13.00 & 352.914004 & -2.701678 & 0.79 & 0.35576 & $55,000.9005$ & 0.99 \\
\hline 60017821 & 13.00 & 355.093408 & -7.796992 & 0.65 & 0.43993 & $54,999.6872$ & 0.99 \\
\hline 60017822 & 11.30 & 352.818348 & -5.371712 & 0.90 & 0.40335 & $54,999.0627$ & 0.99 \\
\hline 60017887 & 10.51 & 352.531675 & 1.434328 & 0.90 & 0.24716 & $54,999.6422$ & $0.99^{\mathrm{a}}$ \\
\hline 60017946 & 17.25 & 357.040958 & -0.532353 & 0.56 & 0.42050 & $54,998.0176$ & 0.99 \\
\hline 60017969 & 19.15 & 356.424312 & 0.439217 & 0.54 & 0.39317 & $55,001.4297$ & 0.99 \\
\hline 60017970 & 15.74 & 351.671617 & 0.795622 & 0.54 & 0.76696 & $54,999.2826$ & 0.69 \\
\hline 60018031 & 18.61 & 0.786213 & 0.130258 & 0.90 & 2.29300 & $55,007.1017$ & 0.99 \\
\hline 60018081 & 13.06 & 353.644290 & -1.326940 & 0.97 & 1.60295 & $54,999.1753$ & 0.99 \\
\hline 60018229 & 12.57 & 1.362500 & 4.806667 & 0.55 & 2.10321 & $55,000.6335$ & 0.99 \\
\hline 60018241 & 12.54 & 356.162500 & -1.810000 & 0.82 & 0.55376 & $54,999.8679$ & 0.99 \\
\hline 60018243 & 13.33 & 359.750000 & -9.526667 & 0.77 & 0.25624 & $55,000.2796$ & 0.99 \\
\hline 60018343 & 10.05 & 2.241575 & 2.945010 & 0.36 & 4.73439 & $54,999.9628$ & 0.99 \\
\hline 60018394 & 10.22 & 354.033199 & -6.232208 & 0.94 & 1.55390 & $55,006.9063$ & 0.98 \\
\hline 60018435 & 10.37 & 5.163989 & -5.143139 & 0.40 & 1.78887 & $55,000.4050$ & 0.99 \\
\hline 60019244 & 14.40 & 359.491080 & -3.689460 & 0.67 & 2.50773 & $54,988.9739$ & 0.99 \\
\hline 60019950 & 14.80 & 354.970700 & 1.983330 & $\ldots$ & $\ldots$ & $55,000.0634$ & $\ldots$ \\
\hline 60020058 & 14.80 & 356.159940 & -8.852300 & 0.38 & 3.22033 & $55,001.2323$ & $0.99^{\mathrm{a}}$ \\
\hline 60020298 & 14.90 & 354.698130 & -7.806650 & 0.29 & 2.36654 & $54,999.0699$ & 0.99 \\
\hline 60021220 & 15.47 & 356.476390 & -0.525299 & 0.55 & 0.69256 & $55,000.4514$ & 0.99 \\
\hline 60021491 & 11.41 & 0.446743 & -3.168466 & 0.41 & 3.71162 & $55,001.6747$ & 0.99 \\
\hline 60021545 & 10.60 & 0.806291 & -3.911404 & & & $55,000.3158$ & \\
\hline 60023653 & 10.33 & 355.034714 & -2.480564 & 0.27 & 2.94483 & $55,000.0656$ & 0.99 \\
\hline 60024040 & 10.62 & 357.762031 & -2.594677 & 0.33 & 1.91961 & $55,000.1132$ & $0.94^{\mathrm{a}}$ \\
\hline 60024244 & 12.22 & 358.906979 & -4.369421 & & & $55,003.9995$ & $\ldots$ \\
\hline 60024523 & 11.04 & 3.678608 & -5.215159 & 0.77 & 0.87101 & $54,999.9486$ & $0.76^{\mathrm{a}}$ \\
\hline
\end{tabular}

${ }^{a}$ Confidence value was renormalized after excluding the primary classification of "misc." 
TABLE 4

Cross-Matched IDENTIFICATIONS For CANDidATE EBs IN K2

\begin{tabular}{|c|c|}
\hline Kepler ID & Objects within $1^{\prime}$ and their SIMBAD classifications \\
\hline $60017809^{\mathrm{a}}$ & 2MASS J23293314-0346078 (Candidate EB*); 1RXS J232933.9-034601 (X) \\
\hline $60017810^{\mathrm{a}}$ & 1SWASP J000437.82+033301.2 (Candidate EB*) \\
\hline $60017812^{\mathrm{a}}$ & 2MASS J00164102-0009251 (low-mass*) \\
\hline $60017814^{\mathrm{a}}$ & $\mathrm{V} *$ EL Aqr $(\mathrm{EB} *$ WUMa) \\
\hline $60017815^{\mathrm{a}}$ & TYC 5255-370-1 (Candidate EB*) \\
\hline $60017816^{\mathrm{a}}$ & 2MASS J23313936-0242060 (Candidate EB*) \\
\hline $60017821^{\mathrm{a}}$ & NSVS 11904371 (Candidate EB*) \\
\hline $60017822^{\mathrm{a}}$ & TYC 5257-616-1 (Candidate EB*); 1RXS J233116.9-052239 (X) \\
\hline 60017887 & 2MASS J23300759+0126037 (pMS*) \\
\hline 60017946 & SDSS J234809.83-003156.4 (low-mass*) \\
\hline 60017969 & SDSS J234541.83+002621.1 (low-mass*) \\
\hline 60017970 & SDSS J232641.19+004744.1 (low-mass*) \\
\hline 60018031 & SDSS J000308.69+000749.0 (low-mass*) \\
\hline 60018081 & $\mathrm{~V}^{*}$ EQ Psc $\left(\mathrm{V}^{*}\right)$ \\
\hline 60018229 & TYC 4-517-1 (Star) \\
\hline 60018241 & NSVS 11906468 (Candidate EB*) \\
\hline 60018243 & $\ldots$ \\
\hline $60018343^{\mathrm{b}}$ & TYC 4-331-1 (Star) \\
\hline 60018394 & BD-07 6054 (Star) \\
\hline 60018435 & BD-05 43 (Star) \\
\hline 60019244 & $\ldots$ \\
\hline 60019950 & .. \\
\hline 60020058 & 2MASS J23443838-0851082 (Star); HD 222891 (Candidate EB*); 1RXS J234438.7-085054 (X) \\
\hline 60020298 & PB $7745($ Star) \\
\hline 60021220 & $\ldots$ \\
\hline 60021491 & TYC 4666-383-1 (Star) \\
\hline 60021545 & TYC 4666-518-1 (Star) \\
\hline 60023653 & BD-03 5686 (Star) \\
\hline 60024040 & TYC 5256-76-1 (Star) \\
\hline 60024244 & TYC 5256-1076-1 (Star) \\
\hline 60024523 & $\ldots$ \\
\hline
\end{tabular}

NotES.—All Simbad object types are defined at http://simbad.u-strasbg.fr/simbad/sim-display?data=otypes. "X" is an X-ray source, "V*" is a variable star, "PMS" is a premain sequence star, and "low-mass*" is a star below solar mass. 2MASS, Two Micron All Sky Survey; 1RXS, 1st ROSAT (ROentgen SATellite) X-ray Survey; 1SWASP, Super Wide Angle Search for Planets; SDSS, Sloan Digital Sky Survey; TYC, Tycho mission; PB, Palomar obs. Berger; NSVS, Northern Sky Variability Survey; BD, Bonner Durchmusterung.

${ }^{a}$ Kepler ID is listed as an EB in K2 engineering target list.

${ }^{b}$ Identified by Poleski et al. (2010; Table 1, line 5) as an SB1 EB with a period of 4.72277 days.

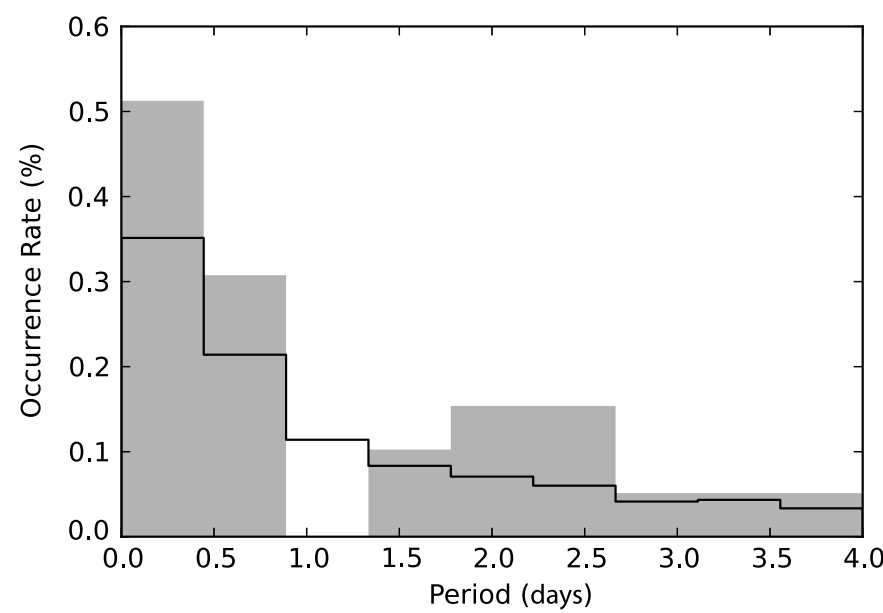

FIG. 4.-Occurrence rate as a function of period for the K2 engineering candidate EBs (gray bars) and EBs from the original Kepler mission (black outline).

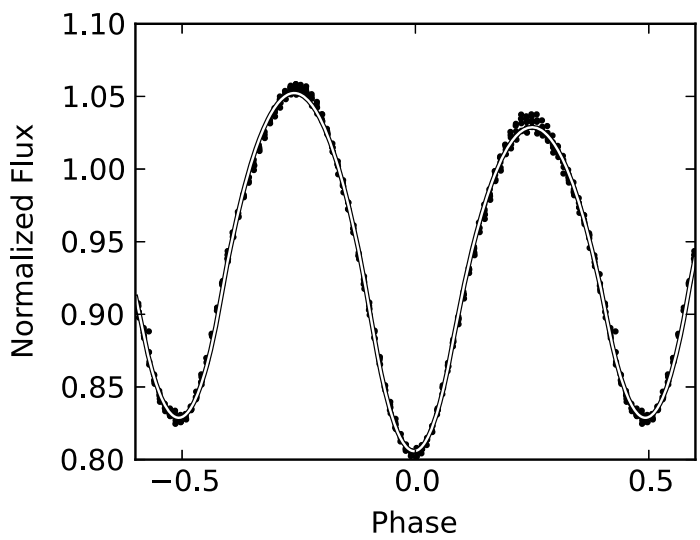

FIG. 5.-Example of the chain of polynomials fit to a phased light curve. These analytic functions are used to quantify the morphology of the potential EB. 


\subsection{EB Ephemerides and Morphologies}

Ephemerides for the candidate EB systems that exhibited at least three subsequent eclipse events are determined by computing a periodogram for each detrended light curve using BLS (Kovács et al. 2002), manually adjusting the correct period if necessary, and setting $\mathrm{BJD}_{0}$ so that the deeper eclipse is placed
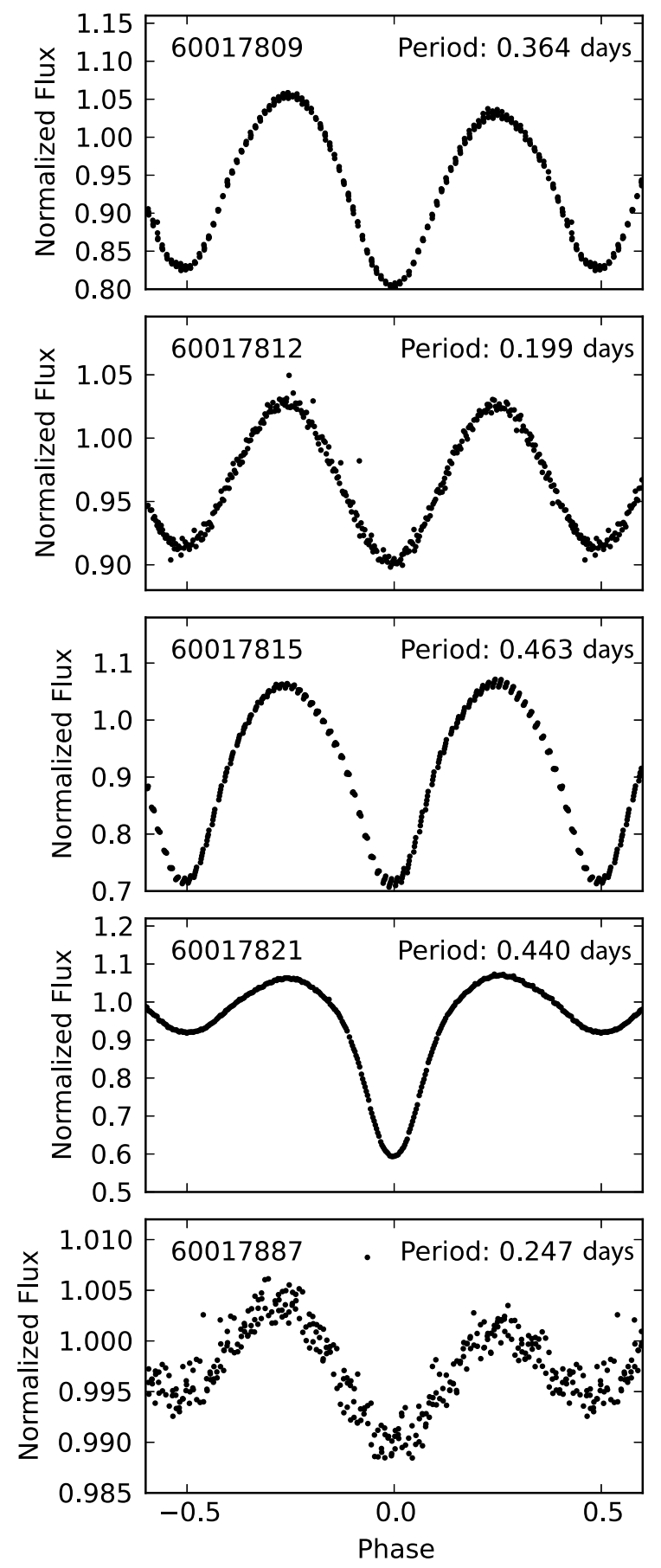

at zero phase. The ephemerides for all 31 candidate $\mathrm{EBs}\left(\mathrm{BJD}_{0}\right.$ for all 31 and periods when possible) are listed in Table 2 and are available online at http://keplerEBs.villanova.edu/k2. Despite such a small sample size, the distribution in EB orbital periods is consistent with that found from the original mission (Fig. 4), with a total detected candidate EB occurrence rate of $1.6 \%$.
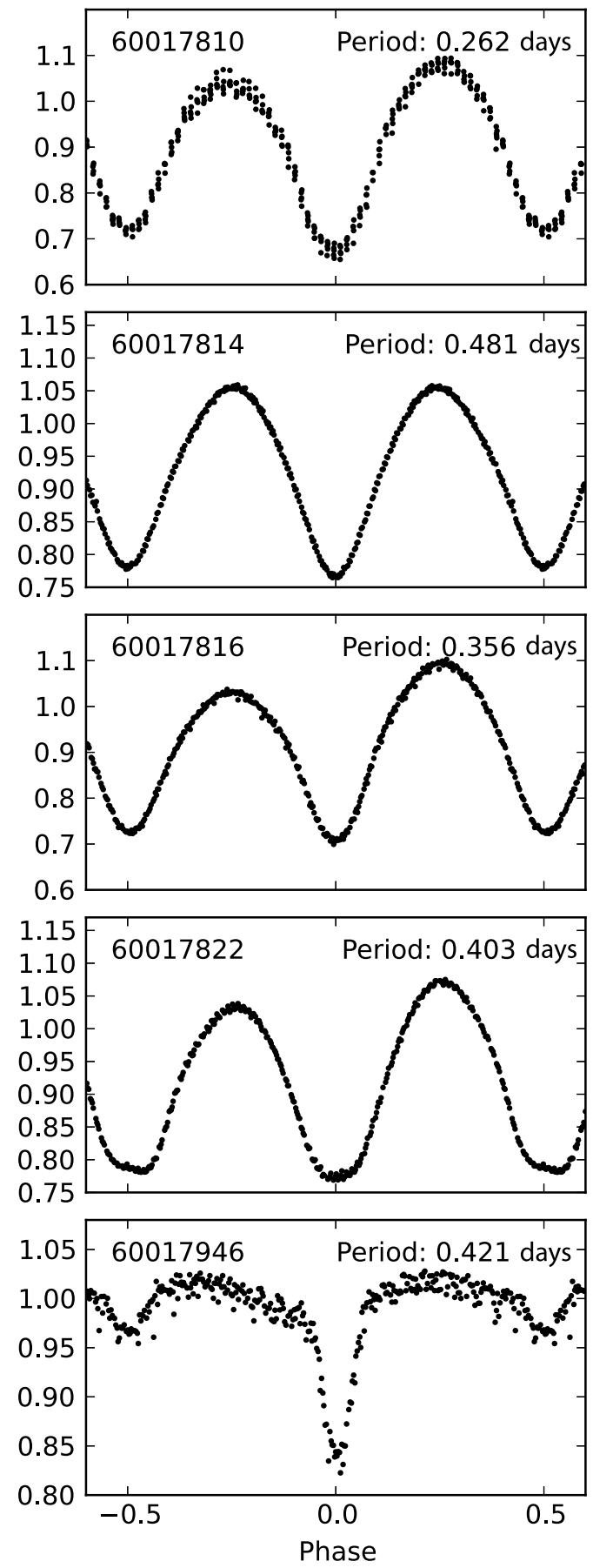
The light curves are phase-folded and fit by a chain of four quadratic functions that describe the shape of the phased light curve (Fig. 5), as described in Prša et al. (2011). This analytic function is then used to determine the morphology, a value between zero (detached) and one (overcontact), using locally linear embedding (Matijevič et al. 2012). These values are listed in Table 2 under the "morph" column. The detrended and phased light curves for all candidate EBs with determined periods are shown in Figure 6, and the standard deviation of the residuals around these analytic functions are listed in Table 1.
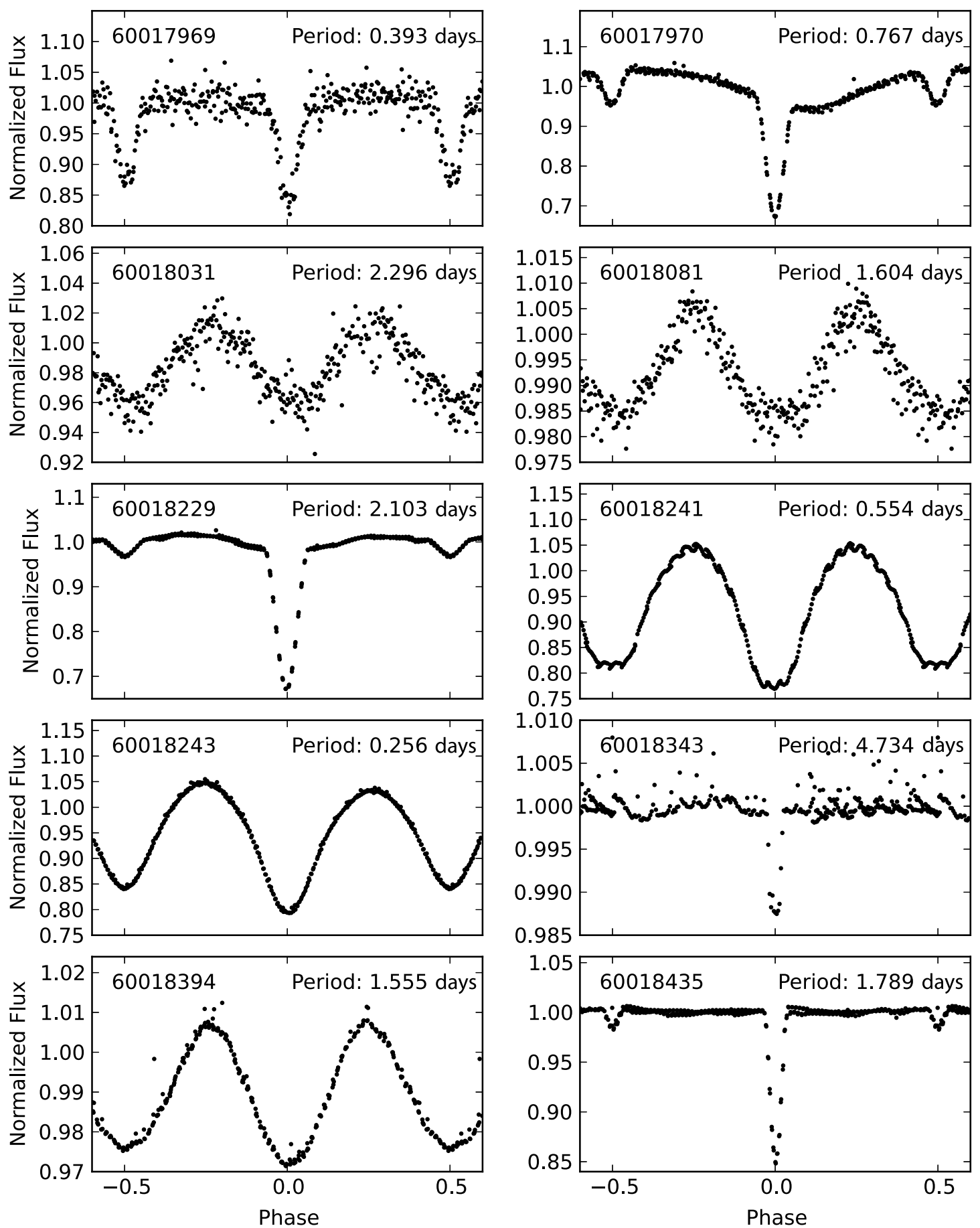

FIG. 6-Continued 


\subsection{Quantitative Assessment of EBs from Automated EB Identification}

The candidate EBs identified in the $\mathrm{K} 2$ dataset provide an initial benchmark set for newly developed pipelines intended for automated discovery of EBs from large datasets such as those that will be provided by the ongoing K2 mission. We applied the Eclipsing Binary Factory (EBF) pipeline (Paegert et al. 2014; Parvizi et al. 2014) to the K2 light curves to test its ability to correctly recover these EBs. The EBF correctly recovered $92 \%$ of the manually identified candidate K2 EBs with at least $90 \%$ confidence in the classification, where the confidence here represents the posterior probability for one of ten variable types derived from priors associated with EBF's neural network
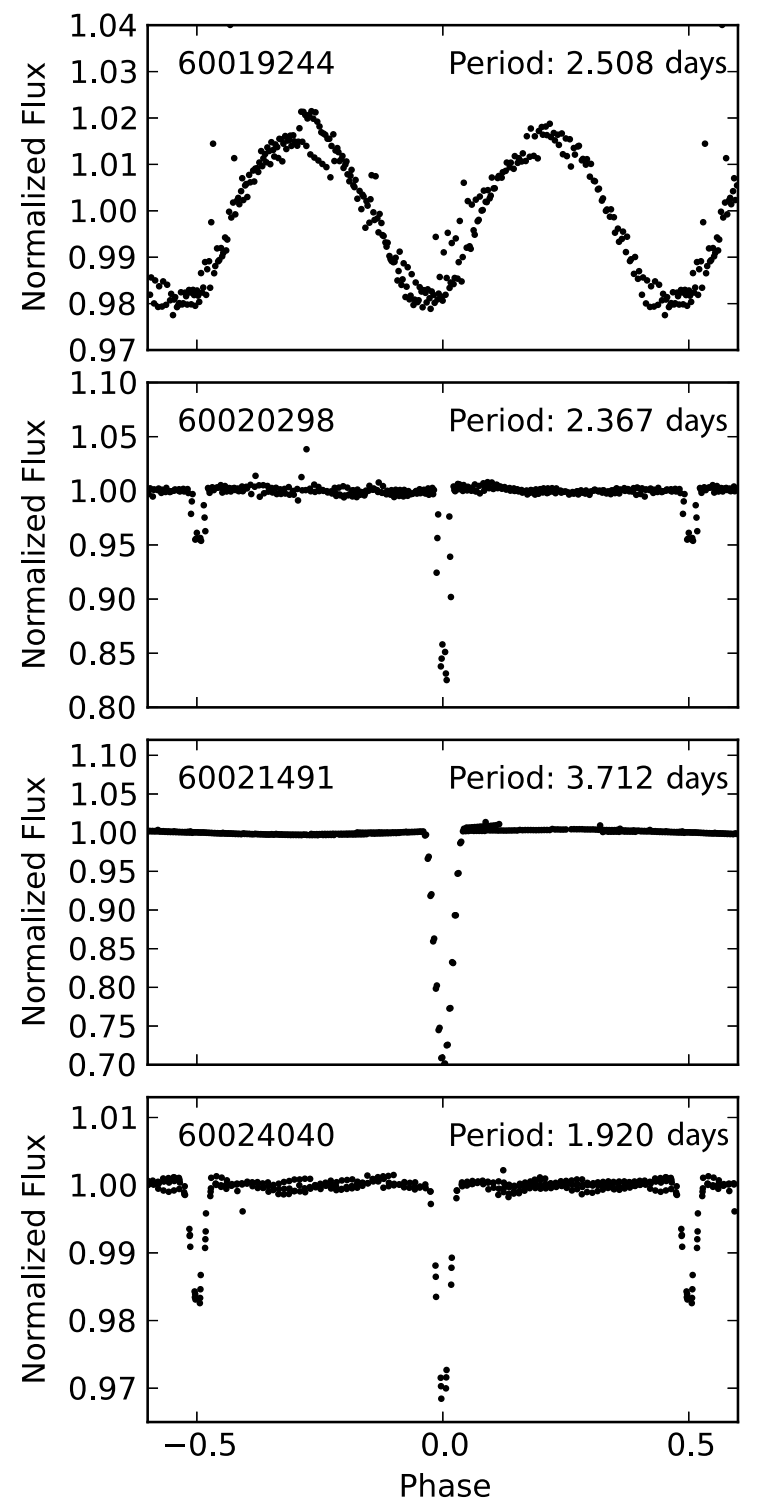

training set (Paegert et al. 2014). This recovery rate is similar to that obtained by the EBF from the original Kepler dataset (Parvizi et al. 2014), suggesting that automated methods such as the EBF are capable of identifying a large sample of EBs in the upcoming K2 campaigns with good completeness.

The probability-based confidence level provided by EBF for each of its output classifications can also be used as a quantitative assessment of the reliability of each EB. Four objects returned a primary classification from EBF of "misc" (miscellaneous), which is a vestige of the original All Sky Automated Survey (ASAS) classification scheme. Since in all of these cases the next most likely classification returned by EBF is "EB," in these four cases we renormalize the confidence level, effectively ignoring the "misc" classification; these classification
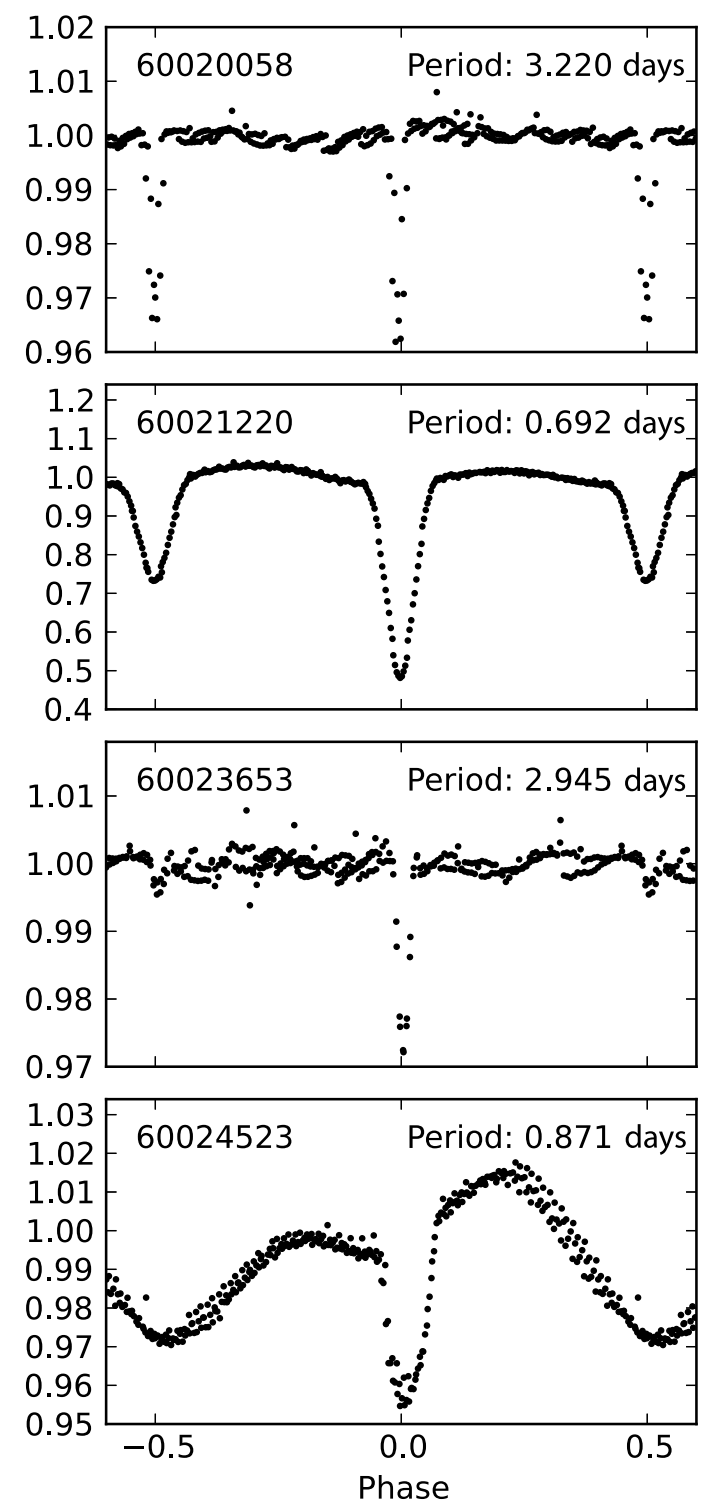

FIG. 6-Continued 
probabilities should be regarded as suggestive. For all of the other candidate EBs (24 cases possessing periods), the EBF classification probabilities cleanly represent the most likely quantitative classification confidence level. These confidence levels are reported in Table 2 and serve as a quantification for how likely each candidate may be a real EB, relative to all the other classes of periodic variable stars recognized by EBF. In all but two cases the confidence level is $\sim 99 \%$, and in the other two cases is $70 \%-80 \%$.

\section{SUMMARY AND DISCUSSION}

Thirty-one candidate eclipsing binaries in the K2 engineering dataset and their ephemerides have been provided when possible. Although the target masks and light-curve extraction process are different than they were in the original Kepler mission, the developed tools are still applicable, and the acquired data are still of high quality for most eclipsing binary science, including all future campaigns of the $\mathrm{K} 2$ mission.

The fraction of candidate EBs identified in the $\mathrm{K} 2$ engineering dataset is $1.6 \%$, in agreement with the fraction of EBs having periods shorter than 5 days in previous Kepler EB studies (Prša et al. 2011; Slawson et al. 2011).
The results of this pilot study show that the K2 light curves are a trove of data for identification, classification, and detailed study of EBs along the ecliptic, which include a number of interesting stellar populations (e.g., large numbers of benchmark clusters of various ages) that were not included in the original Kepler footprint (Prša et al. 2014). Visual identification remains an effective approach to identifying EBs with high completeness. However, approaches such as the EBF pipeline (Paegert et al. 2014) show good promise for fully automating this search and achieving an equivalent level of completeness.

The authors gratefully acknowledge everybody who has made Kepler, and especially the K2 mission, possible. K. E. C. and K. G. S. gratefully acknowledge support from NASA Astrophysical Data Analysis Program grant NNX12AE22G. A. P. gratefully acknowledges support from the NASA Kepler Participating Scientist Program grant NNX12AD20G. S. B. is supported by the Foundation for Fundamental Research on Matter (FOM), which is part of the Netherlands Organisation for Scientific Research (NWO). This research has made use of the SIMBAD database, operated at CDS, Strasbourg, France.

\section{REFERENCES}

$\rightarrow$ Batalha, N. M., et al. 2010, ApJ, 713, L 109

$\rightarrow$ Conroy, K. E., Prša, A., Stassun, K. G., Orosz, J. A., Fabrycky, D. C., \& Welsh, W. F. 2014, AJ, 147, 45

$\rightarrow$ Doyle, L. R., et al. 2011, Science, 333, 1602

$\rightarrow$ Fischer, D. A., et al. 2012, MNRAS, 419, 2900

$\rightarrow$ Howell, S. B., et al. 2014, PASP, 126, 398

$\rightarrow$ Kovćs, G., Zucker, S., \& Mazeh, T. 2002, A\&A, 391, 369

$\rightarrow$ Matijević, G., Prša, A., Orosz, J. A., Welsh, W. F., Bloemen, S., \& Barclay, T. 2012, AJ, 143, 123

Páapics, P. I., et al. 2013, A\&A, 553, A 127 $\rightarrow$ Paegert, M., Stassun, K. G., \& Burger, D. 2014, AJ, 148, 31

Parvizi, M., Paegert, M., \& Stassun, K. G. 2014, AJ, arXiv:1409.3237

$\rightarrow$ Poleski, R., McCullough, P. R., Valenti, J. A., Burke, C. J., Machalek, P., \& Janes, K. 2010, ApJS, 189, 134

Prša, A., Robin, A., \& Barclay, T. 2014, Int. J. Astrobiol., in press

$\rightarrow$ Prša, A., et al. 2011, AJ, 141, 83

$\rightarrow$ Slawson, R. W., et al. 2011, AJ, 142, 160

Vanderburg, A., \& Johnson, J. A. 2014, preprint (arXiv:1408 .3853)

$\rightarrow$ Welsh, W. F., et al. 2012, Nature, 481, 475 